\title{
Accelerated long-term forgetting in healthy older adults predicts cognitive decline over
}

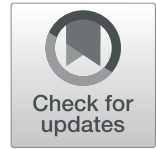
1 year

\author{
Alfie R. Wearn ${ }^{1,2^{*}}$ D, Esther Saunders-Jennings ${ }^{1}$, Volkan Nurdal ${ }^{1,3}$, Emma Hadley $^{1}$, Michael J. Knight ${ }^{4}$,
} Margaret Newson ${ }^{2,4}$, Risto A. Kauppinen ${ }^{3}$ and Elizabeth J. Coulthard ${ }^{1,2}$

\begin{abstract}
Background: Here, we address a pivotal factor in Alzheimer's prevention-identifying those at risk early, when dementia can still be avoided. Recent research highlights an accelerated forgetting phenotype as a risk factor for Alzheimer's disease. We hypothesized that delayed recall over 4 weeks would predict cognitive decline over 1 year better than 30-min delayed recall, the current gold standard for detecting episodic memory problems which could be an early clinical manifestation of incipient Alzheimer's disease. We also expected hippocampal subfield volumes to improve predictive accuracy.
\end{abstract}

Methods: Forty-six cognitively healthy older people (mean age 70.7 $\pm 7.97,21 / 46$ female), recruited from databases such as Join Dementia Research, or a local database of volunteers, performed 3 memory tasks on which delayed recall was tested after 30 min and 4 weeks, as well as Addenbrooke's Cognitive Examination III (ACE-III) and CANTAB Paired Associates Learning. Medial temporal lobe subregion volumes were automatically measured using highresolution $3 \mathrm{~T} \mathrm{MRI}$. The ACE-III was repeated after 12 months to assess the change in cognitive ability. We used univariate linear regressions and ROC curves to assess the ability of tests of delayed recall to predict cognitive decline on ACE-III over the 12 months.

Results: Fifteen of the 46 participants declined over the year ( $\geq 3$ points lost on ACE-III). Four-week verbal memory predicted cognitive decline in healthy older people better than clinical gold standard memory tests and hippocampal MRI. The best single-test predictor of cognitive decline was the 4-week delayed recall on the world list $\left(R^{2}=.123, p=.018, \beta=.418\right)$. Combined with hippocampal subfield volumetry, 4-week verbal recall identifies those at risk of cognitive decline with 93\% sensitivity and 86\% specificity ( $A \cup C=.918, p<.0001$ ).

Conclusions: We show that a test of accelerated long-term forgetting over 4 weeks can predict cognitive decline in healthy older people where traditional tests of delayed recall cannot. Accelerated long-term forgetting is a sensitive, easy-to-test predictor of cognitive decline in healthy older people. Used alone or with hippocampal MRI, accelerated forgetting probes functionally relevant Alzheimer's-related change. Accelerated forgetting will identify early-stage impairment, helping to target more invasive and expensive molecular biomarker testing.

Keywords: Long-term memory, MRI, Hippocampus, Medial temporal lobe, Alzheimer's disease, Early diagnosis

\footnotetext{
* Correspondence: Alfie.wearn@bristol.ac.uk

${ }^{1}$ Bristol Medical School, University of Bristol, Bristol, UK

${ }^{2}$ Institute of Clinical Neurosciences, North Bristol NHS Trust, Bristol, UK

Full list of author information is available at the end of the article
}

\section{$\triangle B M C$}

(c) The Author(s). 2020 Open Access This article is licensed under a Creative Commons Attribution 4.0 International License, which permits use, sharing, adaptation, distribution and reproduction in any medium or format, as long as you give appropriate credit to the original author(s) and the source, provide a link to the Creative Commons licence, and indicate if changes were made. The images or other third party material in this article are included in the article's Creative Commons licence, unless indicated otherwise in a credit line to the material. If material is not included in the article's Creative Commons licence and your intended use is not permitted by statutory regulation or exceeds the permitted use, you will need to obtain permission directly from the copyright holder. To view a copy of this licence, visit http://creativecommons.org/licenses/by/4.0/ The Creative Commons Public Domain Dedication waiver (http://creativecommons.org/publicdomain/zero/1.0/) applies to the data made available in this article, unless otherwise stated in a credit line to the data. 


\section{Background}

Identifying and treating neuropathology leading to Alzheimer's disease $(\mathrm{AD})$ dementia in the early stages, when symptoms are minimal, is the optimal strategy to maintain quality of life. Developing effective treatments for $\mathrm{AD}$ may have been hampered by a failure to detect disease before pathological decline becomes inexorable [1]. Predicting progression at the stage of mild cognitive impairment (MCI) has had some success $[2,3]$. However, in order to identify patients early, we need a marker that can be easily applied at scale with low cost and low patient burden at a stage before patients have symptoms that impair quality of life. If successful in trials, such a marker could be integrated into clinical care to enable early diagnosis and treatment.

Long-term memory over days or weeks may be an effective early marker of AD. Delayed recall over 1 week on a story task has been shown to have a much greater ability to distinguish healthy controls from those with MCI than recall over $30 \mathrm{~min}$ [4]. Another study showed that a delay of 6 weeks on story and complex figure recall tasks could identify significant impairment in a group of people who report 'subjective' memory impairment but who have no objective memory deficit on standard cognitive tests including delayed recall after 30 min [5]. This highlights that people with 'subjective' memory impairment may, in some cases, simply have impairment for which standard tests lack sufficient sensitivity. In a presymptomatic autosomal dominant $\mathrm{AD}$ cohort, Weston et al. [6] demonstrate that long-term memory on a verbal recall test over a 1-week delay not only highlights significant impairment but also predicts years-to-expected-age-of-dementia-onset. Finally, 1-week verbal memory is also impaired in otherwise asymptomatic APOE \&4 homozygotes compared to people with one or no $\varepsilon 4$ alleles [7].

These longer-term memory tests may tax memory circuit functionality more than 30-min delayed recall tests-the current clinical gold standard cognitive task for detecting episodic memory problems which could be an early clinical manifestation of incipient AD. This 'accelerated long-term forgetting' (ALF) phenotype may represent a failure of memory consolidation processes [8] whereby information can be retained for short periods of time, but a successful conversion of labile shortterm memory traces to permanent long-term memory is impossible. Such processes are thought to be primarily governed by the medial temporal lobe (MTL) [9-12], within which ALF may enable earlier identification of dysfunction.

The memory impairment observed in the earliest signs of $\mathrm{AD}$ can be attributed to damage to the hippocampus and MTL cortices. MTL atrophy, measured using magnetic resonance imaging (MRI), is a non-invasive early marker often in routine clinical use [13]. MTL areas affected early in $\mathrm{AD}$ are often reduced in volume even prior to the onset of clinically detectable cognitive symptoms [14].

In the present study, we aimed to test whether delayed recall over 4 weeks is a more sensitive early marker of AD-related cognitive decline than traditional tests of memory over $30 \mathrm{~min}$. We also explored whether MTL subfield volume could complement ALF in identifying early signs of $\mathrm{AD}$.

\section{Methods \\ Participants}

We recruited 53 cognitively healthy community-dwelling older participants with Addenbrooke's Cognitive Examination III (ACE-III) scores of $>88$, the upper threshold for detecting mild cognitive impairment [15]. Forty-six returned for follow-up 1 year later (mean age $70.7 \pm$ 7.97). Twenty-one (46\%) of these participants were female, and participants had $16.2 \pm 3.52$ years of education. Participants were recruited from local volunteer databases, the Join Dementia Research platform and word of mouth. All participants were verbally screened for a history of neurological disorders and memory problems in a telephone interview. All patients provided informed written consent prior to testing. Ethical approval was given by Frenchay NHS Research Ethics Committee.

\section{Cognitive testing}

Cognitive change over the year was assessed using the Addenbrooke's Cognitive Examination III ( $\triangle \mathrm{ACE}-\mathrm{III})$, a freely available 15-min routine test for global cognition with high reliability and utility in dementia diagnosis [15-17]. The ACE-III comprises tests for 5 cognitive domains: attention, memory, language, fluency and visuospatial, and is scored out of a maximum of 100. Multiple versions of the ACE-III are available to allow for retesting. The version assigned to each participant at their baseline visit (either A or B) was randomized. The remaining version was administered at follow-up.

Delayed recall over $30 \mathrm{~min}$ and 4 weeks was tested on three separate tests:

1) Word list-a 16-word list based on the California Verbal Learning Task-II (CVLT-II) [18]; maximum score, 16

2) Story-from the Rivermead Behavioural Memory Test-3 (RBMT-3) [19]; maximum score, 21

3) Complex figure-based on the Rey-Osterrieth Complex Figure Task (RCFT) [20]; maximum score, 36

These tests were adapted from their standard protocols (i.e. that of CVLT-II, RBMT-3 or RCFT) in that participants were trained to $75 \%$ accuracy on each test, with 
2-5 learning trials to equate learning between individuals [21]. Standard CVLT-II procedure involves exactly 5 learning trials, and standard RBMT-3 story protocol involves just one, regardless of performance. Similarly, the RCFT procedure usually incorporates just one copy and one recall test during the learning phase. For the adapted complex figure task, copy and recall trials were both repeated 2-5 times throughout the learning phase. Both delayed recall time points were performed in person, and participants were not told that recall would be tested after 4 weeks.

We took a pragmatic approach in this paper to try and identify the ideal test which would be quick and easy to administer and provide high sensitivity and specificity. Although a single test would be ideal, as it would be feasible to do all 3 of these tests in clinical practice we calculated the composite score (by averaging the scores as a proportion of the maximum possible score for each test) to see if we could increase the sensitivity and specificity at any given testing time point for cognitive decline over a year. A priori (based on data generated by others), we suspected that either word list memory or story memory over 4 weeks would be most sensitive to ADrelated cognitive change.

Further testing included the Paired Associate Learning (PAL) using CANTABeclipse v5 (Cambridge Cognition Ltd.; Cambridge, UK). This test has demonstrated utility in detecting cognitive deficit due to $\mathrm{AD}$ [22-25], so we included it to compare whether our tests could better predict cognitive decline. However, we acknowledge this test is not without its criticisms and limitations [26, 27]. Measures used included total accuracy, mean reaction time and maximum level reached.

\section{Imaging parameters}

All MRI scans were acquired on a Siemens Magnetom Skyra 3T system. The volumetric imaging protocol included a 3D T1-weighted MPRAGE (acquired resolution $0.86 \mathrm{~mm}$ isotropic) and two 2D T2-weighted highresolution hippocampal turbo spin-echo sequences (multi-contrast and single-contrast) (reconstructed resolution $0.34 \times 0.34 \times 1.5 \mathrm{~mm}$ ). Full imaging details are as described by Nurdal et al. [28].

\section{Image analysis}

MTL subfields were demarcated using the automated hippocampal subfield segmentation (ASHS) software (rev103), using the UPENN atlas comprising a mixture of older adults and MCI patients [29]. An example of ASHS output is shown in Fig. 1. All hippocampal masks created as an output of ASHS were visually inspected for quality. In cases where the multi-echo image was either not present or of too poor quality due to movement artefacts, the single-echo TSE was used instead. We have shown in-house that ASHS outputs from either scan type are not significantly different from one another. Three participants were excluded from the volumetric analysis due to poor mask quality. Analysis of individual subfields was performed on CA1, dentate gyrus (DG), subiculum (SUB), entorhinal cortex (EC) and perirhinal cortex (PC). PC was defined as the BA35 label in the UPENN atlas. CA2 and CA3 were included in models combining all subfields. Whole hippocampal volume (CA1-3 + DG + SUB) was also analysed, to highlight the added value of subfield analyses. Volumes were all normalized to intracranial volume.

\section{Statistical analyses \\ Main analyses}

Predictive power of delayed recall scores was performed in two ways: continuous and discrete ('did they decline or not?'). For the former 'continuous' analysis, univariate linear regressions with delayed recall score of a given test as the independent variable and $\triangle \mathrm{ACE}$-III as the outcome variable. For the latter 'discrete' analysis, participants were split into two groups: those whose ACE-III scores declined over the year vs those whose scores did not decline.

This threshold was defined by the reliable change index (RCI) [30], which is calculated using the following formulae:

$$
\begin{aligned}
& \mathrm{SE}=\mathrm{SD}_{\text {norm }} \times \sqrt{2} \times \sqrt{1-r} \\
& \mathrm{RCI}=\mathrm{SE} \times 1.96
\end{aligned}
$$

where $\mathrm{SE}$ is the standard error of change on a test, $\mathrm{SD}_{\text {norm }}$ is the standard deviation of ACE-III score in the normative data (2.7) [15] and $r$ is the reliability of the test, in this case, given as Cronbach's alpha (0.88) [16]. The resulting reliable change index is 2.59 . Therefore, a

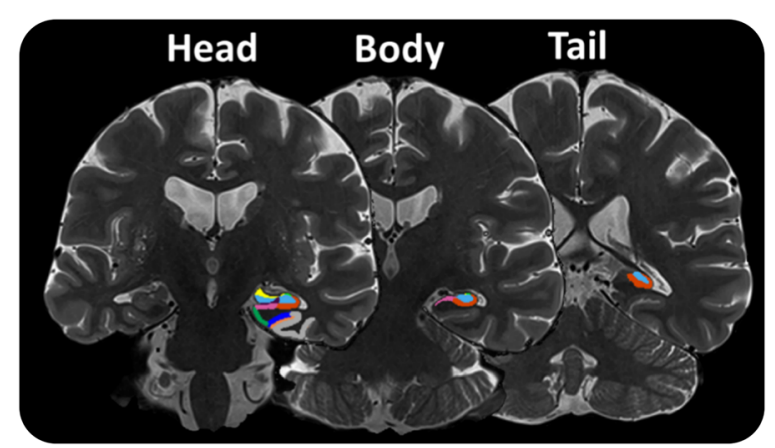

Fig. 1 MTL subfield mask example. Three skull-stripped coronal sections of summed-over-echoes T2-w scan of a single participant are shown with anterior MTL (head) on the left and posterior hippocampus (tail) on the right. Shown are subfields CA1 (red), CA2 (light green), CA3 (yellow), DG (light blue), EC (dark green), BA35 (dark blue) and BA36 (grey) 
decline of 3 or more points was deemed representative of mild but statistically significant cognitive decline.

We used receiver operating characteristic (ROC) curves to assess the predictive capability of each variable in identifying those who declined vs those who did not. Predicted probabilities from multiple logistic regressions were used to create ROC curves of multiple combined variables. ROC Curves were assessed using the area under the curve (AUC) and compared using DeLong's test for dependent ROC curve comparisons [31]. Standard errors (SE) for AUC are shown in the supplementary information (Supplementary Table 2).

\section{Exploratory analysis}

In order to fully assess the added value of MRI volumetry in predicting cognitive decline, we calculated ROC AUC for various combinations of variables (Supplementary Table 2). In order to explore the relationships between different tests of delayed recall, we also calculated Pearson's correlation coefficients for each pairing (supplementary Table 1).

All reported $p$ values are two-tailed $(\alpha=0.05)$, and uncorrected for multiple comparisons given that tests were not fully independent from one another (supplementary Table 1). However, Bonferroni-corrected $p$ values (corrected across all 3 delayed recall tests) are shown where appropriate for full transparency (as $p_{\text {bonferroni }}$ ). All analyses were performed in IBM SPSS Statistics 24, except DeLong tests to compare ROC curves which were carried out in MedCalc v19.4.

\section{Results}

Four-week $(4 \mathrm{w})$ recall on the word list task predicted $\triangle$ ACE-III $\left(R^{2}=.123, p=.018\right.$; $\left.p_{\text {bonferroni }}=.054\right)$ (Table 1 ; Fig. 2). There was no such relationship between $\triangle \mathrm{ACE}-$ III and $30-\mathrm{min}(30 \mathrm{~m})$ recall on the word list $\left(R^{2}=.028\right.$, $\left.p=.274 ; p_{\text {bonferroni }}=.822\right)$. Scores at either time point of the story (30m: $R^{2}=.006, p=.629 ; 4 \mathrm{w} R^{2}=.027, p=.289$ ) and complex figure tasks (30m: $R^{2}=.0003, p=.912$; $4 \mathrm{w}$ : $\left.R^{2}=.045, p=.171\right)$ could not predict $\triangle$ ACE-III. Strikingly, in this healthy cohort, neither whole hippocampal volume nor volume of any single hippocampal subfield or MTL cortical region was correlated with change in performance over the year (Table 1). Furthermore, PAL measures did not predict cognitive decline.

The $4 \mathrm{w}$ composite score significantly positively predicted $\Delta$ ACE-III $\left(R^{2}=.152, p=.013, \beta=.390\right)$, yet $30 \mathrm{~m}$ composite recall score did not $\left(R^{2}=.021, p=.378, \beta=\right.$ .143) (Fig. 2).

Fifteen of the 46 people in this cohort declined more than expected using the reliable change index. Demographic and delayed recall data for decliners and nondecliners are shown in Table 2. Raw cognitive test data for each can be found in supplementary information.
Table 1 Univariate linear regression statistics for delayed recall, PAL scores and MTL region volumes predicting $\triangle A C E-I I I$

\begin{tabular}{lllll}
\hline Predictors & $\boldsymbol{\beta}$ (standardized) & $\boldsymbol{R}^{\mathbf{2}}$ & $\boldsymbol{p}$ & Number \\
\hline Word List & & & & \\
30m & .167 & .028 & .274 & 45 \\
$4 \mathrm{~W}$ & .350 & .123 & .018 & 45 \\
Story & & & & \\
30m & .076 & .006 & .629 & 43 \\
4W & .165 & .027 & .289 & 43 \\
Complex figure & & & & \\
30m & .018 & .0003 & .912 & 42 \\
4w & .246 & .061 & .116 & 42 \\
PAL & & & & \\
Accuracy & .194 & .037 & .225 & 41 \\
Reaction time & -.021 & .0004 & .898 & 41 \\
Max level & .064 & .004 & .689 & 41 \\
Whole hippocampus & -.072 & .005 & .646 & 43 \\
CA1 & -.084 & .007 & .590 & 43 \\
DG & -.059 & .003 & .707 & 43 \\
SUB & .005 & $<.0001$ & .976 & 43 \\
EC & .098 & .010 & .532 & 43 \\
PC & .173 & .030 & .269 & 43 \\
\hline
\end{tabular}

Both 30m and 4w word list recall scores distinguished decliners from non-decliners. In line with our hypothesis, the $4 \mathrm{w}$ recall time point had the strongest discriminatory power for a single variable with an AUC of .752 $(\mathrm{SE}=.078, p=.007) .30 \mathrm{~m}$ verbal recall $(\mathrm{AUC}=.687, \mathrm{SE}=$ $.080 p=.047$ ), and $30 \mathrm{~m}$ (story: $\mathrm{AUC}=.699, \mathrm{SE}=.083$, $p=.040$; complex figure: $\mathrm{AUC}=.497, \mathrm{SE}=.099, p=.979$ ) and $4 \mathrm{w}$ measures (story: $\mathrm{AUC}=.671, \mathrm{SE}=.083, p=.079$; complex figure: $\mathrm{AUC}=.625, \mathrm{SE}=.090, p=.191$ ) in other tests had weaker discriminatory power. The $4 \mathrm{w}$ composite recall score was able to predict ACE-III decline status (AUC $=.761, \mathrm{SE}=.076, p=.008)$, whereas the $30 \mathrm{~m}$ composite score was less effective (AUC $=.674, \mathrm{SE}=.085$, $p=.078)$. Comparing the ROC curves using the Delong method [31] did not reveal statistically significant differences between $30 \mathrm{~m}$ and $4 \mathrm{w}$ AUCs for any test (word list: $p=.472$; story: $p=.785$; complex figure: $p=.437$; composite: $p=.369$ ).

Combining memory test scores with MTL regional volumes improves classification. $4 \mathrm{w}$ word list recall combined with individual subfield volumes produced the best single-test model with an AUC of .918 $(p<.0001$; Fig. 3). The optimal cut-off has $93 \%$ sensitivity and $86 \%$ specificity. The equivalent model using $30 \mathrm{~m}$ word list recall score achieved an AUC of $.829(p=.001)$. In comparison, MTL subfields alone produced a predictive 

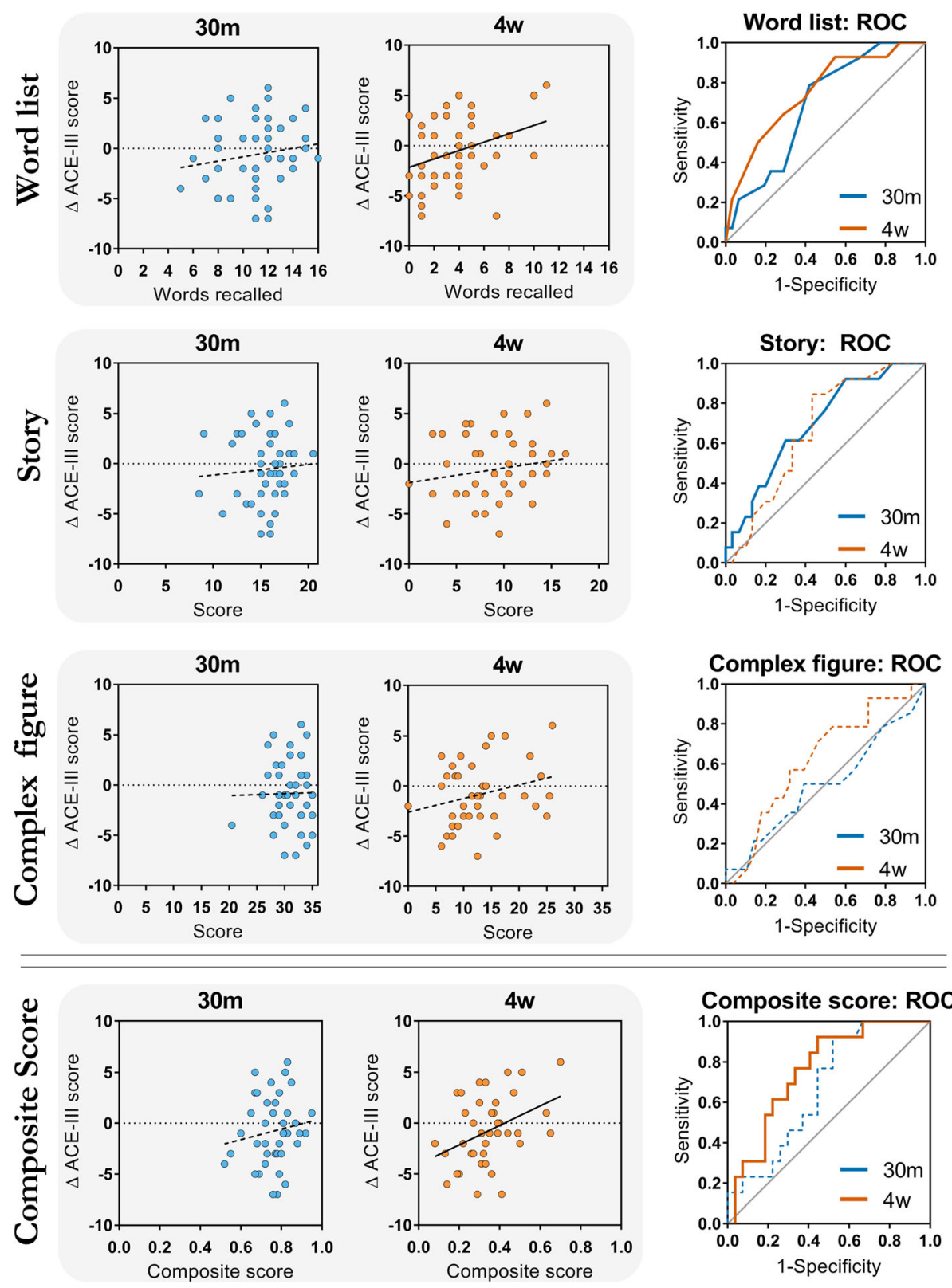

Fig. 2 Delayed recall predicting cognitive decline over 1 year: linear regressions (left) and ROC curves (right). Linear regression panels (left) show raw data for each test and $\triangle$ ACE-III. Smaller scores on a 4-week word list recall indicate a more negative score change. Solid lines represent statistically significant regressions/ROC curves $(p<.05)$. Dotted lines represent non-significant regressions/ROC curves $(p>.05)$. 30m, 30-min recall scores; 4w, 4-week recall scores; ACE-III, Addenbrooke's Cognitive Examination III

model with an AUC of $.802(p=.001)$. All ROC data can be seen in supplementary Table 2 .

\section{Discussion}

We report that extending the delay period over which recall, particularly verbal recall, is tested from $30 \mathrm{~min}$ to 4 weeks can markedly improve the identification of people likely to decline cognitively in the coming year. This is the first time this has been shown in a longitudinal cohort study. ALF improves classification accuracy beyond that which is achieved using MTL subfield volumes. These findings support the clinical utility of much longer-term memory tests in identifying the very subtle neuropathology that occurs years before a diagnosis of $\mathrm{AD}$ dementia [32], though further research is required to fully validate their use in predicting conversion to dementia.

Previous work in a selective presymptomatic cohort with the extremely rare autosomal dominant form of Alzheimer's disease suggested that ALF might be a 
Table 2 Demographic information and delayed recall data for people who did or did not decline over the year

\begin{tabular}{llll}
\hline & Non-decliner & Decliner & Total \\
\hline N (male:female) & $31(16: 15)$ & $15(9: 6)$ & $46(25: 21)$ \\
Age (years) & $71.1 \pm 8.92$ & $69.9 \pm 5.74$ & $70.7 \pm 7.97$ \\
YOE & $16.7 \pm 3.41$ & $15.1 \pm 3.61$ & $16.2 \pm 3.35$ \\
ACE-III /100 & & & \\
$\quad$ Baseline & $95.0 \pm 3.03$ & $94.7 \pm 3.20$ & $94.9 \pm 3.05$ \\
$\quad$ Follow-up & $96.1 \pm 2.73$ & $90.5 \pm 3.14$ & $94.2 \pm 3.88$ \\
Word list /16 & & & \\
$\quad 30 m$ & $11.5 \pm 2.55$ & $9.73 \pm 2.37$ & $10.9 \pm 2.61$ \\
$\quad 4 w$ & $4.35 \pm 2.79$ & $2.14 \pm 2.03$ & $3.67 \pm 2.76$ \\
Story /21 & & & \\
30m & $16.0 \pm 2.22$ & $14.2 \pm 2.49$ & $15.4 \pm 2.42$ \\
4w & $9.70 \pm 4.07$ & $7.65 \pm 2.85$ & $9.08 \pm 3.83$ \\
Complex figure /36 & & & \\
30m & $31.0 \pm 2.57$ & $30.2 \pm 4.07$ & $30.7 \pm 3.15$ \\
4w & $13.5 \pm 6.42$ & $11.5 \pm 4.95$ & $12.9 \pm 5.99$ \\
\hline
\end{tabular}

Data are shown as mean \pm standard deviation. Maximum scores for each cognitive test are shown in the left column

YOE years of education, 30m 30-min delayed recall time point, $4 \mathrm{w}$ 4-week delayed recall time point

sensitive marker of early change despite having normal scores after a 30-min delay [6]. They tested all three of a story, word list and complex figure task over a delay of 1 week, and only on the word list was longer-term memory worse in genetically at-risk participants. Here, we utilized a real-world sample to reveal that long-term word list memory has the potential to be applied to the general older population as a marker of future cognitive

\section{Subfield volumes + verbal recall}

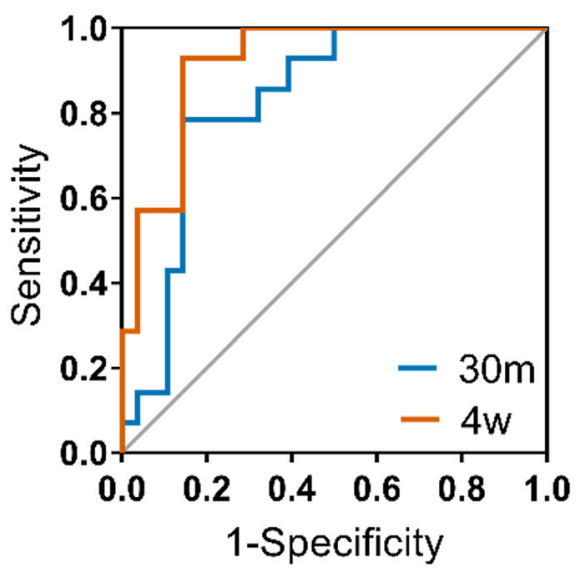

Fig. 3 Accuracy of delayed verbal recall and MTL subfield volumes predicting cognitive decline. Shown are receiver operating characteristic (ROC) curves for individual combined subfields (CA1-3, DG, EC, BA35) and either $30-\mathrm{min}(30 \mathrm{~m})$ or 4-week $(4 \mathrm{w})$ recall on the word list decline. These complementary findings suggest that the word list may present the best single test to predict atrisk patient groups and that story learning or visuospatial memory tasks may be less sensitive. Alignment of our results with those from a different study in a cohort genetically at-risk is encouraging as to the reliability of this finding. Future clinical trials could utilize memory tests over a longer time frame as a sensitive marker of change. It should be noted that the 4-week verbal recall test predicting cognitive change over the year was not below the $\alpha$ threshold of statistical significance after a Bonferroni correction $(p=.054)$ across the 3 cognitive tests used. However, the Bonferroni method is a highly conservative method of correcting for multiple comparisons, and given that the three tests cannot be regarded as fully independent (as shown in supplementary Table 1), we believe this still to be a robust, albeit preliminary, finding. Future work should aim to confirm or deny this finding.

ROC curves for word list recall show that very high levels of sensitivity are achievable with small trade-offs in specificity. For example, a cut-off score of 4.5 at the $4 \mathrm{w}$ time point allows $93 \%$ of people who decline by more than three points on ACE-III to be detected, even though $55 \%$ of people below this cut-off do not decline by more than this amount. This enables the test to be used as a wide-net screening tool, the main goal of which is to reduce the number of people having more expensive and invasive testing for probable $A D$, such as CSF analyses or amyloid/tau PET scans [33]. A 4-week memory task takes only a few minutes to administer and could transform standard clinical neuropsychology testing (where delayed recall of a word list over $30 \mathrm{~min}$ is usually administered). This test could even be adapted to be performed over the phone or delivered by a mobile app, to reduce face-to-face clinic visits. There is the caveat of having to wait 4 weeks to get any result from this test. However, although we used a 4-week delay paradigm in this study, we do not show that it is necessary to wait this long to measure ALF. Indeed, shorter delays are less susceptible to confounding from the variation in events between delay time points. The delay period in past work ranges from 1 to 6 weeks [4-6], but further work is required to determine the optimal delay that is sensitive to cognitive dysfunction, but least burdensome for participants and clinicians.

Given the critical role for MTL in long-term memory consolidation [34], ALF may reflect subtle MTL damage, such as that which occurs in the prodromal stages of AD. In combination with MTL subregion volumes, 4week verbal recall scores predict cognitive decline in cognitively healthy individuals with sensitivity and specificity similar to that of much more expensive molecular biomarkers tests (PET scanning and CSF analysis) [35- 
37]. The present analyses provide further justification for the incorporation of MRI and automated volumetry techniques in clinical diagnostic processes [13, 38, 39]. As neuroimaging, often MRI, is part of routine clinical screening processes for neurological disease, the combination of cognitive tests with volumetry is highly practical and easily translatable. It is interesting to note that although MTL volumes are able to predict the presence or absence of cognitive decline (in our ROC analysis), they were very poor in predicting any linear association with cognitive change. This may indicate a non-linear relationship between the two, whereby only MTL values below a certain threshold are indicative of cognitive decline, and cognitive change in people with larger MTL volumes is more determined by other factors (such as tissue damage that has not yet resulted in volume change detectable on MRI [40, 41]). This supports theories of graceful degradation and cognitive reserve [42] and should be further explored in studies with larger sample sizes. ALF may also require the functionality of networks beyond the MTL that are still affected in early $\mathrm{AD}$, such as those involving the thalamus [43].

It could be suggested that the observed ALF phenotype is explained by the differences in practice effects between individuals. Lack of practice effects (failure to improve performance when a task is repeated) has been shown to be predictive of cognitive decline in people with amnestic mild cognitive impairment [44]. This seems relatively unlikely to be the case here as task instructions requirements were conceptually very simplefree recall tests, rather than re-administration of any learning materials. Furthermore, during the learning phase, differences in individual learning rates were controlled for by including the $75 \%$ accuracy threshold, above which learning stimuli were not repeated. We therefore believe an attenuated practice effect to be a distinct measure, albeit potentially with similar neurobiological underpinnings, whereby impaired memory consolidation circuits may impair an individual's ability to benefit from repeated trials.

\section{Limitations}

A limitation of the present study is that the global cognitive decline seen over the year cannot be directly attributed to AD without assessment of amyloid and/or tau status via CSF or PET. This was not collected as part of the present study but is an area of future interest and presents an obvious next step for this research. Despite this, we suggest that tests of delayed recall may predict cognitive decline that is specifically due to AD pathology given the role of MTL in long-term memory consolidation $[34,45]$ and the susceptibility of MTL to early AD pathology. Furthermore, as discussed, this study is highly in line with the findings of Weston et al. [6], which directly links delayed recall over days/weeks to autosomal dominant $\mathrm{AD}$.

Although the 4-week recall time point of the word list task produced the greatest AUC for any single cognitive test in detecting which people experienced cognitive decline, the differences between time points were relatively small. This is in part due to the high correlations between test time points, at least for the story and word list (supplementary Table 1); however, this is also due to our relatively small sample size. A larger sample size would be required to confirm that differences in classification ability are statistically significant, as well as to improve the certainty of conclusions from all conducted analyses. Future studies may use our findings to assist in calculating sample sizes required for sufficient power to detect such statistical differences.

Throughout this paper, we discuss mild cognitive decline defined as a statistically reliable change in score on the ACE-III, a test of general cognitive function. Although a drop of just 3 points is statistically significant, it may not be clinically significant in terms of a functional decline and is certainly not necessarily indicative of a decline to a level of MCI. Given the group (healthy older adults), sample size (46) and follow-up period of this study (1 year), a subtle measure of cognitive change was necessary, as opposed to measuring conversion to $\mathrm{MCI}$ or $\mathrm{AD}$. We infer that a subtle cognitive decline over this timescale may indicate further cognitive decline over longer timescales. In this regard, the marker does not necessarily need to be directly clinically functionally significant, but rather, it would herald the need for further investigation to detect the cause of the change and, where possible, avoid a functionally significant change. Further follow-ups of these participants would be necessary to observe which people decline further or even which people later receive diagnoses of dementia. Further research is also required to explicitly test the responsiveness of the ACE-III to detecting subtle cognitive decline.

Another limitation of our study is the validity of the cognitive tests used to measure delayed recall. Although based on well-validated tasks designed to measure recall at $30 \mathrm{~min}$, we modified the protocols slightly (e.g. by including a learning criterion and varying the number of learning trials). In doing so, there was a risk that we invalidated the tests for measuring delayed recall at $30 \mathrm{~min}$. Although the data are not comparable to normative data of the tests on which they are based, we believe they still provide robust measures of delayed recall at both time points as we observed substantial variation in scores at both time points in all 3 tests, indicating a lack of floor and ceiling effects. 
Finally, it could be argued that an incorporation bias exists, given that the cohort was defined as healthy by having an ACE-III $>88,26$ points of which are achieved from tests of memory. We do not believe this to be a factor which biases our results, as memory is tested over delays of no more than 10 min during the ACE-III, and this forms a small part of the entire ACE-III score (the 10 -min delayed recall test is worth just 7 points in total). Even if the cohort was defined solely on having normal 30-min recall at baseline, this could not be expected to influence whether 30-min or 4-week memory would better predict cognitive decline over the year.

\section{Conclusions}

In summary, we show that a test of accelerated longterm forgetting over 4 weeks can predict cognitive decline in healthy older people where traditional tests of delayed recall cannot. This highlights its potential in screening patients for clinical trials and practice either in conjunction with volumetry or as a standalone test. This could reduce the number of patients who need expensive biomarker testing for Alzheimer's disease, increasing the feasibility of large-scale early clinical trials. Further research is required to explore the relationship between ALF and molecular biomarker profiles to define the specificity of longer-term recall deficits to early $\mathrm{AD}$ pathology.

\section{Supplementary information}

Supplementary information accompanies this paper at https://doi.org/10. 1186/s13195-020-00693-4.

Additional file 1. Supplementary tables.

\section{Acknowledgements}

The authors wish to thank Join Dementia Research and the Avon \& Wiltshire Mental Health Partnership for their assistance with the participant recruitment. We also wish to thank those who have helped collect data for the project (Ellen Gaaikema, Lucy Adams, Candida Stainer, Ben Kershaw) as well as all the volunteers who gave up their time to take part in our study.

\section{Authors' contributions}

This manuscript was written by A.W. and E.C. The cognitive testing protocol was designed by A. W, E.C. and M.N. Imaging protocols were set up by R. K, and M.K. M.K. also set up imaging analysis pipelines which were later managed by A.W. Cognitive testing, scoring and imaging were carried out by A.W., V.N., E.S-J. and E.H. The authors read and approved the final manuscript.

\section{Funding}

This study was funded by Alzheimer's Research UK, BRACE and Wellcome (109067/Z/15/Al).

\section{Availability of data and materials}

The datasets used and/or analysed during the current study are available from the corresponding author on reasonable request.

\section{Ethics approval and consent to participate}

All patients provided informed written consent prior to testing. Ethical approval was given by the Frenchay NHS Research Ethics Committee.
Consent for publication

Not applicable

\section{Competing interests}

We declare that none of the authors has competing financial or nonfinancial interests.

\section{Author details}

${ }^{1}$ Bristol Medical School, University of Bristol, Bristol, UK. ${ }^{2}$ Institute of Clinical Neurosciences, North Bristol NHS Trust, Bristol, UK. ${ }^{3}$ Department of Psychology, University of Bath, Bath, UK. ${ }^{4}$ School of Psychological Science, University of Bristol, Bristol, UK.

Received: 20 May 2020 Accepted: 16 September 2020

Published online: 28 September 2020

\section{References}

1. Marsden G, Mestre-Ferrandiz J. Dementia: the R\&D landscape. Office of Health Economics: Research; 2015.

2. Vecchio F, Miraglia F, Iberite F, Lacidogna G, Guglielmi V, Marra C, et al. Sustainable method for Alzheimer dementia prediction in mild cognitive impairment: electroencephalographic connectivity and graph theory combined with apolipoprotein E. Ann Neurol. 2018;84(2):302-14.

3. Marizzoni M, Ferrari C, Jovicich J, Albani D, Babiloni C, Cavaliere L, et al. Predicting and tracking short term disease progression in amnestic mild cognitive impairment patients with prodromal Alzheimer's disease: structural brain biomarkers. J Alzheimers Dis. 2019;69(1):3-14.

4. Walsh CM, Wilkins S, Bettcher BM, Butler CR, Miller BL, Kramer JH. Memory consolidation in aging and $\mathrm{MCl}$ after 1 week. Neuropsychology. 2014;28(2):273-80.

5. Manes F, Serrano C, Calcagno ML, Cardozo J. Accelerated forgetting in subjects with memory complaints. J Neurol. 2008;255(7):1067-70.

6. Weston PSJ, Nicholas JM, Henley SMD, Liang Y, Macpherson K, Donnachie E, et al. Accelerated long-term forgetting in presymptomatic autosomal dominant Alzheimer's disease: a cross-sectional study. Lancet Neurol. 2018; 17(2):123-32.

7. Zimmermann JF, Butler CR. Accelerated long-term forgetting in asymptomatic APOE $\varepsilon 4$ carriers. Lancet Neurol. 2018;17(5):394-5.

8. Hoefeijzers S, Dewar M, Sala SD, Zeman A, Butler C. Accelerated long-term forgetting in transient epileptic amnesia: an acquisition or consolidation deficit? Neuropsychologia. 2013;51(8):1549-55.

9. Eichenbaum H. A cortical-hippocampal system for declarative memory. Nat Rev Neurosci. 2000;1 (1):41-50.

10. Scoville WB, Milner B. Loss of recent memory after bilateral hippocampal lesions. J Neurol Neurosurg Psychiatry. 1957;20(1):11-21.

11. Alvarez P. Squire LR. Memory consolidation and the medial temporal lobe: a simple network model. Proc Natl Acad Sci. 1994;91(15):7041-5.

12. Squire $L R$, Zola-Morgan $S$. The medial temporal lobe memory system. Science. 1991;253(5026):1380-6.

13. Apostolova LG, Mosconi L, Thompson PM, Green AE, Hwang KS, Ramirez A, et al. Subregional hippocampal atrophy predicts Alzheimer's dementia in the cognitively normal. Neurobiol Aging. 2010;31(7):1077-88.

14. Barnes J, Bartlett JW, van de Pol LA, Loy CT, Scahill RI, Frost C, et al. A metaanalysis of hippocampal atrophy rates in Alzheimer's disease. Neurobiol Aging. 2009:30(11):1711-23.

15. Hsieh S, Schubert S, Hoon C, Mioshi E, Hodges JR. Validation of the Addenbrooke's cognitive examination III in frontotemporal dementia and Alzheimer's disease. Dement Geriatr Cogn Disord. 2013;36(3-4):242-50.

16. Noone P. Addenbrooke's cognitive examination-III. Occup Med. 2015;65(5): $418-20$.

17. Bruno D, Vignaga SS. Addenbrooke's cognitive examination III in the diagnosis of dementia: a critical review. Neuropsych Dis Treat. 2019;15: $441-7$.

18. Delis DC, Kramer JH, Kaplan E, Ober BA. California verbal learning testsecond edition (CVLT-II). San Antonio: Psychological Corporation; 2000.

19. Wilson B, Cockburn J, Baddeley A, Hiorns R. The development and validation of a test battery for detecting and monitoring everyday memory problems. J Clin Exp Neuropsychol. 1989;11(6):855-70.

20. Shin M-S, Park S-Y, Park S-R, Seol S-H, Kwon JS. Clinical and empirical applications of the Rey-Osterrieth Complex Figure Test. Nature Protocols. 2006; (2):nprot.2006.115. 
21. Geurts S, van der Werf SP, Kessels RPC. Accelerated forgetting? An evaluation on the use of long-term forgetting rates in patients with memory problems. Front Psychol. 2015;6:752.

22. Fowler KS, Saling MM, Conway EL, Semple JM, Louis WJ. Paired associate performance in the early detection of DAT. J Int Neuropsychol Soc. 2002; 8(1):58-71.

23. Barnett JH, Blackwell AD, Sahakian BJ, Robbins TW. The paired associates learning (PAL) test: 30 years of CANTAB translational neuroscience from laboratory to bedside in dementia research. Curr Top Behav Neurosci. 2016; 28:449-74.

24. Kuzmickienè J, Kaubrys G. Selective ability of some CANTAB battery test measures to detect cognitive response to a single dose of donepezil in Alzheimer disease. Med Sci. 2015;21:2572-82.

25. Égerházi A, Berecz R, Bartók E, Degrell I. Automated Neuropsychological Test Battery (CANTAB) in mild cognitive impairment and in Alzheimer's disease. Prog Neuro-Psychopharmacol Biol Psychiatry. 2007;31(3):746-51.

26. Gonçalves MM, Pinho MS, Simões MR. Test-retest reliability analysis of the Cambridge Neuropsychological Automated Tests for the assessment of dementia in older people living in retirement homes. Appl Neuropsychol Adult. 2015;23(4):251-63.

27. Smith PJ, Need AC, Cirulli ET, Chiba-Falek O, Attix DK. A comparison of the Cambridge Automated Neuropsychological Test Battery (CANTAB) with "traditional" neuropsychological testing instruments. J Clin Exp Neuropsychol. 2013;35(3):319-28.

28. Nurdal V, Wearn A, Knight M, Kauppinen R, Coulthard E. Prospective memory in prodromal Alzheimer's disease: real world relevance and correlations with cortical thickness and hippocampal subfield volumes. Neurolmage. 2020;26:102226.

29. Yushkevich PA, Pluta JB, Wang H, Xie L. Automated volumetry and regional thickness analysis of hippocampal subfields and medial temporal cortical structures in mild cognitive impairment. Hum Brain Mapp. 2015;36(1):256-87.

30. Jacobson NS, Truax P. Clinical significance: a statistical approach to defining meaningful change in psychotherapy research. J Consult Clin Psychol. 1991; 59(1):12.

31. DeLong ER, DeLong DM, DLJB C-P. Comparing the areas under two or more correlated receiver operating characteristic curves: a nonparametric approach; 1988. p. 837-45.

32. Bateman RJ, Xiong C, Benzinger TLS, Fagan AM, Goate A, Fox NC, et al. Clinical and biomarker changes in dominantly inherited Alzheimer's disease. N Engl J Med. 2012;367(9):795-804.

33. Harten AC, Visser PJ, YAL P, Teunissen CE, Blankenstein MA, Scheltens $P$ et al. Cerebrospinal fluid $A \beta 42$ is the best predictor of clinical progression in patients with subjective complaints. Alzheimers Dement. 2013;9(5):481-7.

34. Squire LR, Genzel L, Wixted JT, Morris RG. Memory consolidation. Cold Spring Harbor Perspectives in Biology. 2015;7(8):a021766.

35. Hansson $O$, Zetterberg H, Buchhave P, Londos E, Blennow K, Minthon L. Association between CSF biomarkers and incipient Alzheimer's disease in patients with mild cognitive impairment: a follow-up study. Lancet Neurol. 2006;5(3):228-34.

36. Hertze J, Minthon L, Zetterberg H, Vanmechelen E, Blennow K, Hansson O. Evaluation of CSF biomarkers as predictors of Alzheimer's disease: a clinical follow-up study of 4.7 years. J Alzheimers Dis. 2010;21(4):1119-28.

37. Ma Y, Zhang S, Li J, Zheng D-M, Guo Y, Feng J, et al. Predictive accuracy of amyloid imaging for progression from mild cognitive impairment to Alzheimer disease with different lengths of follow-up. Medicine. 2014;93(27):e150.

38. Apostolova LG, Dutton RA, Dinov ID, Hayashi KM, Toga AW, Cummings JL, et al. Conversion of mild cognitive impairment to Alzheimer disease predicted by hippocampal atrophy maps. Arch Neurol. 2006;63(5):693-9.

39. Chételat G, Fouquet M, Kalpouzos G, Denghien I, la Sayette DV, Viader F, et al. Three-dimensional surface mapping of hippocampal atrophy progression from $\mathrm{MCl}$ to $\mathrm{AD}$ and over normal aging as assessed using voxel-based morphometry. Neuropsychologia. 2008;46(6):1721-31.

40. Knight MJ, Wearn A, Coulthard E, Kauppinen RA. T2 relaxometry and diffusion tensor indices of the hippocampus and entorhinal cortex improve sensitivity and specificity of MRI to detect amnestic mild cognitive impairment and Alzheimer's disease dementia. J Magn Reson Imaging. 2019:49(2):445-55
41. Wearn AR, Nurdal V, Saunders-Jennings E, Knight MJ, Isotalus HK, Dillon S, Tsivos D, Kauppinen RA, Coulthard EJ. T2 heterogeneity: a novel marker of microstructural integrity associated with cognitive decline in people with mild cognitive impairment. Alzheimers Res Ther. 2020;12(1).

42. Stern Y. Cognitive reserve. Neuropsychologia. 2009;47(10):2015-28.

43. Aggleton JP, Pralus A, Nelson AJD, Hornberger M. Thalamic pathology and memory loss in early Alzheimer's disease: moving the focus from the medial temporal lobe to Papez circuit. Brain. 2016;139(7):1877-90.

44. Duff K, Lyketsos CG, Beglinger LJ, Chelune G, Moser DJ, Arndt S, et al. Practice effects predict cognitive outcome in amnestic mild cognitive impairment. Am J Geriatric Psychiatry. 2011;19(11):932-9.

45. Saloner R, Casaletto KB, Marx G, Dutt S, Bussche VAB, You M, et al. Performance on a 1-week delayed recall task is associated with medial temporal lobe structures in neurologically normal older adults. Clin Neuropsychol. 2017;32(3):1-12.

\section{Publisher's Note}

Springer Nature remains neutral with regard to jurisdictional claims in published maps and institutional affiliations.

Ready to submit your research? Choose BMC and benefit from:

- fast, convenient online submission

- thorough peer review by experienced researchers in your field

- rapid publication on acceptance

- support for research data, including large and complex data types

- gold Open Access which fosters wider collaboration and increased citations

- maximum visibility for your research: over $100 \mathrm{M}$ website views per year

At $\mathrm{BMC}$, research is always in progress.

Learn more biomedcentral.com/submissions 